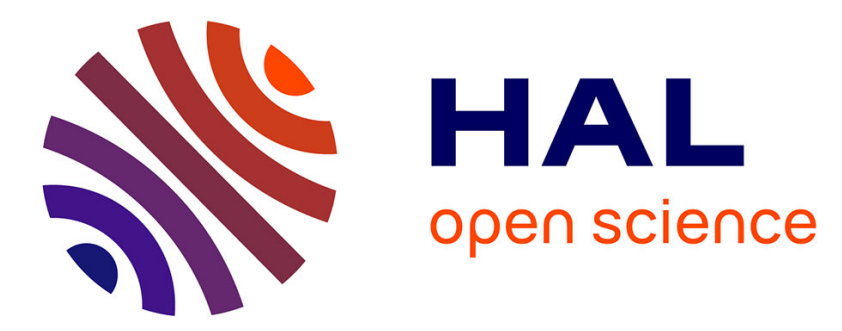

\title{
An Integrated Control Strategy in Disturbance Decoupling of Max-Plus Linear Systems with Applications to a High Throughput Screening System in Drug Discovery
}

\author{
Ying Shang, Laurent Hardouin, Mehdi Lhommeau, Carlos-Andrei Maia
}

\section{To cite this version:}

Ying Shang, Laurent Hardouin, Mehdi Lhommeau, Carlos-Andrei Maia. An Integrated Control Strategy in Disturbance Decoupling of Max-Plus Linear Systems with Applications to a High Throughput Screening System in Drug Discovery. 53rd IEEE Conference on Decision and Control, 2014, Los Angeles, United States. pp.6. hal-01113455

\author{
HAL Id: hal-01113455 \\ https://hal.science/hal-01113455
}

Submitted on 5 Feb 2015

HAL is a multi-disciplinary open access archive for the deposit and dissemination of scientific research documents, whether they are published or not. The documents may come from teaching and research institutions in France or abroad, or from public or private research centers.
L'archive ouverte pluridisciplinaire HAL, est destinée au dépôt et à la diffusion de documents scientifiques de niveau recherche, publiés ou non, émanant des établissements d'enseignement et de recherche français ou étrangers, des laboratoires publics ou privés. 


\title{
An Integrated Control Strategy in Disturbance Decoupling of Max-Plus Linear Systems with Applications to a High Throughput Screening System in Drug Discovery
}

\author{
Ying Shang, Laurent Hardouin, Mehdi Lhommeau, and Carlos Andrey Maia
}

\begin{abstract}
This paper presents an integrated control strategy solving disturbance decoupling of max-plus linear systems. The classical disturbance decoupling problem (DDP) in geometric control theory means that the controlled outputs will not be changed by any disturbances. The new proposed modified disturbance decoupling problem (MDDP) ensures that the controlled output signals will not delayed more than the existing delays caused by the disturbances in order to achieve the justin-time optimal control. Furthermore, this paper presents the integration of state-feedback and open-loop control strategies to solve the MDDP, as well as the DDP. If these controls can only solve the MDDP, but not the DDP, an evaluation principle is established to compare the distance between the output signals generated by the controllers and the output signals generated by the disturbances. The main results of this paper are illustrated by a high throughput screening system in drug discovery.
\end{abstract}

\section{INTRODUCTION}

Max-plus linear systems ([1], [10]) are used to model for timed discrete-event systems, which represent the system operations as discrete sequences of events in time. The main advantage of max-plus linear systems is incorporating the traditional linear system theory for the nonlinear concurrent behaviors in discrete-event systems. Over the past three decades, many fundamental problems for max-plus linear systems have been studied by researchers, for example, controllability ([18]), observability ([7]), and model reference control ([15]). However, the geometric theory for maxplus linear systems introduced in ([4]) has not been well established as the traditional linear systems ([2], [21]). Only a few existing research results on generalizing fundamental concepts and problems in the geometric control are generalized to max-plus linear systems, such as computation of different controlled invariant sets ([9], [14], [16]) and the disturbance decoupling problem ([12]).

This paper reports upon further investigations on the disturbance decoupling problem (DDP) for max-plus linear systems, which means that the output signals remain unchanged in the presence of the disturbances. For max-plus linear systems, the controller can only increase the inputs; i.e., to delay the input dates of tokens in the corresponding timed-event graph (TEG), which is a subclass of timed Petri net models for discrete-event systems in which all places have a single transition upstream and a single one downstream. For a manufacturing system, solving the DDP means that the outputs will be delayed more than the delays caused by the disturbances. From a practical point of view, it would be interesting to ask the question as whether there exists a controller such that the system is not disturbed more than the delays caused by the disturbances. Therefore, the modified disturbance decoupling problem (MDDP) in ([8],

Y. Shang is with the Department of Electrical and Computer Engineering, Southern Illinois University Edwardsville, Campus Box 1801, Edwardsville, IL 62026, USA yshange siue.edu

L. Hardouin is with the Laboratorie d'Ingénierie des Systèmes Automatisés of the University of Angers, 62 avenue Notre Dame du Lac, 49000 Angers, France laurent. hardouinduniv-angers.fr

M. Lhommeau is with the Laboratorie d'Ingénierie des Systèmes Automatisés of the University of Angers, 62 avenue Notre Dame du Lac, 49000 Angers, France mehdi. Ihommeauduniv-angers.fr

C.A. Maia is with Departamento de Engenharia Elétrica, Universidade Federal de Minas Gerais (UFMG). Av. Antônio Carlos 6627, Pampulha, 31270-010, Belo Horizonte, MG, Brazil ma i a a cpdee . u fmg . br. C.A. Maia is grateful to CNPq, FAPEMIG and CAPES for supporting this research
[20]) is to find controls such that the output signals generated by the control will not be greater than the output signals caused by the disturbances. This paper presents an integration of the state-feedback controls and the open-loop controls such that the MDDP can be solved. If these controls can only solve the MDDP, but not the DDP, then an evaluation principle is established to compare the distance, which is interpreted as the event delays between the output signals generated by the controllers and the output signals generated by the disturbances. The main results are illustrated by a high throughput screening system in drug discovery.

\section{Mathematical PREliminaries}

Definition 1: A semiring is a set $\mathcal{S}$, equipped with two operations $\oplus$ and $\otimes$, such that $(\mathcal{S}, \oplus)$ is a commutative monoid (the zero element will be denoted $\varepsilon$ ), $(\mathcal{S}, \otimes)$ is a monoid (the unit element will be denoted $e$ ), operation $\otimes$ is right and left distributive over $\oplus$, and $\varepsilon$ is absorbing for the product (i.e. $\varepsilon \otimes a=a \otimes \varepsilon=\varepsilon, \forall a$ ).

A semiring $\mathcal{S}$ is idempotent if $a \oplus a=a$ for all $a \in \mathcal{S}$. In an idempotent semiring $\mathcal{S}$, operation $\oplus$ induces a partial order relation $a \succeq b \Longleftrightarrow a=a \oplus b, \forall a, b \in \mathcal{S}$. Then, $a \vee b=a \oplus b$. An idempotent semiring $\mathcal{S}$ is complete if the sum of infinite numbers of terms is always defined, and if multiplication distributes over infinite sums too. In particular, the sum of all the elements of the idempotent semiring is denoted $T$ (for "top"). In this paper, we denote $\overline{\mathbb{Z}}_{\max }=$ $(\mathbb{Z} \cup\{-\infty,+\infty\}, \max ,-\infty,+, 0)$, where $\varepsilon=-\infty$ is the identity element to $\max$ and $e=0$ is the identity element to + . the integer max-plus semiring. A non empty subset $\mathcal{B}$ of a semiring $\mathcal{S}$ is a subsemiring of $\mathcal{S}$ if for all $a, b \in \mathcal{B}$ we have $a \oplus b \in \mathcal{B}$ and $a \otimes b \in \mathcal{B}$.

Definition 2: A mapping $f: \mathcal{S} \rightarrow \mathcal{S}$, where $\mathcal{S}$ is a complete idempotent semiring, is residuated if and only if $f(\varepsilon)=\varepsilon$ and $f$ is lower-semicontinuous, that is,

$$
f\left(\bigoplus_{i \in I} a_{i}\right)=\bigoplus_{i \in I} f\left(a_{i}\right),
$$

for any (finite or infinite) set $I$. The mapping $f$ is said to be residuated and $f^{\sharp}$ is called its residual. When $f$ is residuated, $f^{\sharp}$ is the unique order preserving mapping such that

$$
f \circ f^{\sharp} \preceq \mathrm{Id} \quad f^{\sharp} \circ f \succeq \mathrm{Id},
$$

where Id is the identity mapping from $\mathcal{S}$ to $\mathcal{S}$.

It is straightforward that $: L_{a}: \mathcal{S} \rightarrow \mathcal{S}, x \mapsto a x$ and $R_{a}: \mathcal{S} \rightarrow \mathcal{S}, x \mapsto x a$ are lower semi-continuous. Therefore these mappings are both residuated i.e., $L_{a}(x) \preceq b$ (resp. $R_{a}(x) \preceq b$ ) admits a greatest solution, then the following notations are considered :

$$
\begin{aligned}
& L_{a}^{\sharp}(b)=a ф b=\bigoplus\{x \mid a x \preceq b\} \text { and } \\
& R_{a}^{\sharp}(b)=b \phi a=\bigoplus\{x \mid x a \preceq b\}, \quad \forall a, b \in \mathcal{S} .
\end{aligned}
$$

All these results admit a natural extension to the matrix case, where the sum and product of matrices are defined with the same rules than in classical theory (see [1]). Over a complete max-plus algebra, the implicit equation $x=a x \oplus b$ admits $x=a^{*} b$ as the least solution, where $a^{*}=\oplus_{i \in \mathbb{N}} a_{i}$ (Kleene star operator) with $a_{0}=e$. 


\section{Disturbance Decoupling IN MAX-Plus LineAR} SYSTEMS

A max-plus linear system can be described the following

$$
\begin{aligned}
x(k) & =A x(k-1) \oplus B u(k) \oplus S q(k), \\
y(k) & =C x(k),
\end{aligned}
$$

where the state is $x(k) \in X \cong \overline{\mathbb{Z}}_{\max }^{n}$, the input is $u(k) \in U \cong$ $\overline{\mathbb{Z}}_{\text {max }}^{p}$, the disturbance is $q(k) \in Q \cong \overline{\mathbb{Z}}_{\max }^{r}$, and the output is $y(k) \in Y \cong \overline{\mathbb{Z}}_{\max }^{q}$, for $k \in \mathbb{Z}$. This kind of system makes it possible to describe the behaviors of TEGs, by associating to each transition $x_{i}$ a firing date sequence $x_{i}(k) \in \overline{\mathbb{Z}}_{\max }$, and predict the system evolution thanks to Eq. (2).

Definition 3: System (2) is called disturbance decoupled by a state-feedback controller $u(k)=F x(k-1) \oplus v(k))$ (or an open-loop controller $u(k)=P v(k)$ if and only if any disturbance signal will not affect the system output $y(k)$ for all $k \in \mathbb{Z}$, that is, the output signals $y(k)$ remain the same as the output signals of the undisturbed system, for all $k \in \mathbb{Z}$.

In manufacturing systems, for example, when the system breaks down for one hour, the control will delay the system operation more than one hour in order to achieve the DDP. In practical scenarios, production lines need to resume as soon as the system breakdown is fixed. Hence, a modified DDP in the next subsection is introduced in order to find the optimal just-in-time controls such that the system will start running as soon as possible once the system breakdown is recovered.

Definition 4: The max-plus linear system described in Eq. (2) is called modified disturbance decoupled by a state feedback control $u(k)=F x(k-1) \oplus v(k)$ (or an open-loop control $u(k)=P v(k))$ if and only if the system output signals generated by the controls will not be greater than the output signals generated by only the disturbances.

The MDDP means that, for manufacturing systems, the controls will delay the starting dates of the process such that the finishing date of the output parts would be sooner than the finishing dates after the system breakdown. The next section will present the event-domain approach to solve both the DDP and the MDDP.

\section{SOLVING DDP AND MDDP USING EVENT-DOMAIN APPROACH}

\section{A. Event-Domain Representation}

For a state equation in Eq. (2), each increasing sequence $\{x(k)\}$, it is possible to define the transformation $X(\gamma)=$ $\bigoplus x(k) \gamma^{k}$ where $\gamma$ is a backward shift operator in event domain (i.e., $Y(\gamma)=\gamma X(\gamma) \Longleftrightarrow\{y(k)\}=\{x(k-1)\}$, (see [1], p. 228). This transformation is analogous to the $z$ transform used in discrete-time classical control theory and the formal series $X(\gamma)$ is a synthetic representation of the trajectory $x(k)$. The set of the formal power series in $\gamma$ is denoted by $\overline{\mathbb{Z}}_{\max } \llbracket \gamma \rrbracket$ and constitutes an idempotent semiring. Therefore, the state equation in Eq. (2) becomes a polynomial equation or a event-domain representation,

$$
\begin{aligned}
X(\gamma) & =\bar{A} X(\gamma) \oplus B U(\gamma) \oplus S Q(\gamma), \text { where } \bar{A}=\gamma A \\
Y(\gamma) & =C X(\gamma)
\end{aligned}
$$

where the state $X(\gamma) \in\left(\overline{\mathbb{Z}}_{\max } \llbracket \gamma \rrbracket\right)^{n}$, the output $Y(\gamma) \in$ $\left(\overline{\mathbb{Z}}_{\max } \llbracket \gamma \rrbracket\right)^{q}$, the input $U(\gamma) \in\left(\overline{\mathbb{Z}}_{\max } \llbracket \gamma \rrbracket\right)^{p}$, and the disturbance $Q(\gamma) \in\left(\overline{\mathbb{Z}}_{\max } \llbracket \gamma \rrbracket\right)^{r}$, and matrices $\bar{A} \triangleq \gamma A \in\left(\overline{\mathbb{Z}}_{\max } \llbracket \gamma \rrbracket\right)^{n \times n}$, $B \in\left(\overline{\mathbb{Z}}_{\max } \llbracket \gamma \rrbracket\right)^{n \times p}, C \in\left(\overline{\mathbb{Z}}_{\max } \llbracket \gamma \rrbracket\right)^{q \times n}$ and $S \in\left(\overline{\mathbb{Z}}_{\max } \llbracket \gamma \rrbracket\right)^{n \times r}$ represent the link between transitions. According to the state equation (3), the evolution of the system is

$$
\begin{aligned}
X(\gamma) & =\bar{A}^{*} B U(\gamma) \oplus \bar{A}^{*} S Q(\gamma) \\
Y(\gamma) & =C \bar{A}^{*} B U(\gamma) \oplus C \bar{A}^{*} S Q(\gamma) .
\end{aligned}
$$

The trajectories $U(\gamma)$ and $Y(\gamma)$ can be related ([1], p. 243) by the equation $Y(\gamma)=H(\gamma) U(\gamma)$, where $H(\gamma)=$ $C \bar{A}^{*} B \in\left(\overline{\mathbb{Z}}_{\max } \llbracket \gamma \rrbracket\right)^{q \times p}$ is called the transfer matrix of the TEG. Entries of matrix $H$ are periodic series ([1], $\mathrm{p}$. 260) in the idempotent semiring, usually represented by $p(\gamma) \oplus q(\gamma)\left(\tau \gamma^{\nu}\right)^{*}$, where $p(\gamma)$ is a polynomial representing the transient behavior, $q(\gamma)$ is a polynomial corresponding to a pattern which is repeated periodically, the period being given by the monomial $\left(\tau \gamma^{\nu}\right)$. The disturbances are uncontrollable inputs acting on the system internal's state, which model events that block the system, e.g. machine breakdown, uncontrollable component supply through matrix $S$, and $C \bar{A}^{*} S \in\left(\overline{\mathbb{Z}}_{\max } \llbracket \gamma \rrbracket\right)^{q \times r}$ is the transfer function between the disturbances and outputs.

\section{B. Formulation of DDP and MDDP in Event-Domain}

The objective of the MDDP is to find the greatest openloop or state-feedback control $U(\gamma)$ such that the output trajectories will not be disturbed more than the disturbance signals have acted on the system. For example, if a manufacturing system encounters a service breakdown, the control $U(\gamma)$ will delay the input of parts as much as possible to avoid congestion inside of the system, while the system outputs will remain the same as before. Formally, according to Definition 4, this means to find the greatest control, $U(\gamma)$, such that the following equation holds,

$$
\begin{array}{r}
C \bar{A}^{*} B U(\gamma) \oplus C \bar{A}^{*} S Q(\gamma)=C \bar{A}^{*} S Q(\gamma) \\
\Longleftrightarrow C \bar{A}^{*} B U(\gamma) \preceq C \bar{A}^{*} S Q(\gamma) .
\end{array}
$$

According to Definition 3, solving the DDP in event-domain means that the control $U(\gamma)$ has to achieve

$$
\begin{array}{r}
C \bar{A}^{*} B U(\gamma) \oplus C \bar{A}^{*} S Q(\gamma)=C \bar{A}^{*} B U(\gamma) \\
\Longleftrightarrow C \bar{A}^{*} S Q(\gamma) \preceq C \bar{A}^{*} B U(\gamma) .
\end{array}
$$

In this paper, the disturbance is assumed to be measurable and the control architecture is given in Fig. 1. The goal is to find $U(\gamma)=\bar{F} X(\gamma) \oplus V(\gamma)$ such that the MDDP is solved, where $\bar{F}=\gamma F$ if $u(k)=F x(k-1) \oplus v(k)$ and such an $\bar{F}$ can be generalized to a general feedback matrix $F(\gamma)$ consisting $\gamma^{d}$ with $d \geq 1$.

Mathematically, the state and output signals in the event $\gamma$-domain are represented as follows:

$$
\begin{aligned}
X(\gamma) & =(\bar{A} \oplus B \bar{F})^{*} B V(\gamma) \oplus(\bar{A} \oplus B \bar{F})^{*} S Q(\gamma) \\
& =(\bar{A} \oplus B \bar{F})^{*}[B \mid S]\left(\begin{array}{l}
V(\gamma) \\
Q(\gamma)
\end{array}\right) \\
& =(\bar{A} \oplus B \bar{F})^{*} \widetilde{B}\left(\begin{array}{l}
V(\gamma) \\
Q(\gamma)
\end{array}\right), \text { where } \widetilde{B}=[B \mid S], \\
Y(\gamma) & =C X(\gamma)=C(\bar{A} \oplus B \bar{F})^{*} \widetilde{B}\left(\begin{array}{l}
V(\gamma) \\
Q(\gamma)
\end{array}\right) .
\end{aligned}
$$

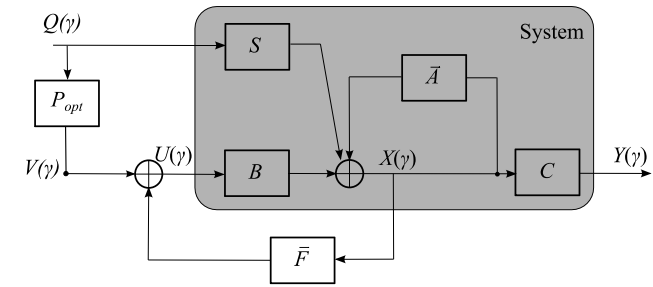

Fig. 1: The controller structure for DDP and MDDP. 
Based on Definition 3, solving the DDP in event-domain means that the state feedback controller has to achieve the following equality:

$$
C(\bar{A} \oplus B \bar{F})^{*} \widetilde{B}\left(\begin{array}{l}
V(\gamma) \\
Q(\gamma)
\end{array}\right)=C(\bar{A} \oplus B \bar{F})^{*} B V(\gamma) .
$$

Based on Definition 4, solving the MDDP in event-domain means that the state feedback controller has to achieve another equality:

$$
C(\bar{A} \oplus B \bar{F})^{*} \widetilde{B}\left(\begin{array}{l}
V(\gamma) \\
Q(\gamma)
\end{array}\right)=C(\bar{A} \oplus B \bar{F})^{*} S Q(\gamma)
$$

Equations (10) and (11) each have three variables, the state feedback structure $\bar{F}$, the open-loop controller $V(\gamma)$, as well as the disturbance input $Q(\gamma)$. If we need the pair of $\bar{F}$ and $V(\gamma)$ to solve the MDDP and the DDP for any arbitrary disturbances, then we can proceed by fixing the open-loop control first and then find state feedback control, or vice versa.

\section{Finding the Integrated Control Strategy by Residuation}

This subsection will find the pre-filter matrix $P$ first, and then find the state feedback matrix $\bar{F}$ by residuation. By considering $F=\varepsilon$ and by the residuation theory(see [20]), we showed that the MDDP is solved if and only if the equation $C \bar{A}^{*} B U(\gamma) \oplus C \bar{A}^{*} S Q(\gamma)=C \bar{A}^{*} S Q(\gamma)$ holds and the greatest solution solving the equation above is

$$
\begin{aligned}
P_{o p t} & \triangleq\left(C \bar{A}^{*} B\right) \phi\left(C \bar{A}^{*} S\right) \\
& =\bigoplus_{P \in \overline{\mathbb{Z}}_{\max } \llbracket \gamma \rrbracket^{p \times r}}\left\{C \bar{A}^{*} B P \preceq C \bar{A}^{*} S\right\},
\end{aligned}
$$

i.e. such a $P_{\text {opt }}$ solves the MDDP for any disturbance $Q(\gamma)$.

Once the open-loop control $V(\gamma)$ is fixed, then we can obtain the state feedback $\bar{F}$ in equations (10) and (11) in order to solve the MDDP and the DDP. The goal is to find a state feedback control $U(\gamma)=\bar{F} X(\gamma) \oplus V(\gamma)$, such that the output signals are the same as the output signals controlled by any open-loop controller $V(\gamma)$. In summary, that is, the following equality holds

$$
C(\bar{A} \oplus B \bar{F})^{*} \widetilde{B}\left(\begin{array}{l}
V(\gamma) \\
Q(\gamma)
\end{array}\right)=C \bar{A}^{*} \widetilde{B}\left(\begin{array}{l}
V(\gamma) \\
Q(\gamma)
\end{array}\right)
$$

Proposition 1: ([11], [13]) The greatest controller $\bar{F}_{\text {opt }}$ is given by

$$
\bar{F}_{\text {opt }}=\left(C \bar{A}^{*} B\right) \phi\left(C \bar{A}^{*} \widetilde{B}\right) \phi\left(\bar{A}^{*} \widetilde{B}\right),
$$

such that the output trajectories generated by the state feedback controller are the same as the output trajectories generated by the open-loop controller, i.e. the equality $C\left(\bar{A} \oplus B \bar{F}_{\text {opt }}\right)^{*} \widetilde{B}=C \bar{A}^{*} \widetilde{B}$ holds.

Proposition 2: The integrated control law $U(\gamma)=$ $\bar{F}_{\text {opt }} X(\gamma) \oplus P_{\text {opt }} Q(\gamma)$ solves the MDDP of the max-plus linear system in Eq. (3), where the open-loop control is $V(\gamma)=P_{o p t} Q(\gamma)=\left(C \bar{A}^{*} B\right) \phi\left(C \bar{A}^{*} S\right) Q(\gamma)$ and the feedback control matrix is $\bar{F}_{\text {opt }}=\left(C \bar{A}^{*} B\right) \phi\left(C \bar{A}^{*} \widetilde{B}\right) \phi\left(\bar{A}^{*} \widetilde{B}\right)$.

Proof: If we apply the integrated control law $U(\gamma)=$ $\bar{F}_{\text {opt }} X(\gamma) \oplus P_{\text {opt }} Q(\gamma)$ to the max-plus linear system in Eq. (3), the following inequalities hold:

$$
\begin{aligned}
& C\left(\bar{A} \oplus B \bar{F}_{o p t}\right)^{*} \widetilde{B}\left(\begin{array}{l}
V(\gamma) \\
Q(\gamma)
\end{array}\right)=C \bar{A}^{*} B V(\gamma) \oplus C \bar{A}^{*} S Q(\gamma) \\
= & C \bar{A}^{*} B P_{o p t} Q(\gamma) \oplus C \bar{A}^{*} S Q(\gamma) \preceq C \bar{A}^{*} S Q(\gamma) \\
\preceq & C\left(\bar{A} \oplus B \bar{F}_{o p t}\right)^{*} S Q(\gamma) .
\end{aligned}
$$

Clearly, the last term is less than the first term in the equation above. Hence, we can achieve the following equality

$$
\begin{array}{r}
C\left(\bar{A} \oplus B \bar{F}_{\text {opt }}\right)^{*} B V(\gamma) \oplus C\left(\bar{A} \oplus B \bar{F}_{\text {opt }}\right)^{*} S Q(\gamma)= \\
C\left(\bar{A} \oplus B \bar{F}_{\text {opt }}\right)^{*} S Q(\gamma) .
\end{array}
$$

Therefore, such an integrated control $U(\gamma)$ solves the MDDP.

Theorem 1 ([20]): The optimal pre-filter $V(\gamma)=$ $P_{\text {opt }} Q(\gamma)$, which solves the MDDP, also solves the DDP for the max-plus linear systems described in Eq. (3) if and only if $\operatorname{Im} C \bar{A}^{*} S \subset \operatorname{Im} C \bar{A}^{*} B$.

Proposition 3: The integrated control law $U(\gamma)=$ $\bar{F}_{\text {opt }} X(\gamma) \oplus P_{\text {opt }} Q(\gamma)$ solves the DDP of the max-plus linear system in Eq. (3) if and only if $\operatorname{Im} C \bar{A}^{*} S \subset$ $\operatorname{Im} C \bar{A}^{*} B$, where the open-loop control is $V(\gamma)=P_{\text {opt }} Q(\gamma)=$ $\left(C \bar{A}^{*} B\right) \phi\left(C \bar{A}^{*} S\right) Q(\gamma)$ and the feedback control matrix is $\bar{F}_{\text {opt }}=\left(C \bar{A}^{*} B\right) \phi\left(C \bar{A}^{*} \widetilde{B}\right) \phi\left(\bar{A}^{*} \widetilde{B}\right)$.

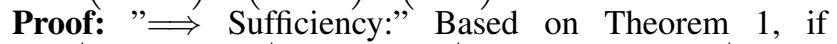
Im $C \bar{A}^{*} S \subset \operatorname{Im} C \bar{A}^{*} B$, then $C \bar{A}^{*} B P_{\text {opt }} Q(\gamma)=C \bar{A}^{*} S Q(\gamma)$ for any $Q(\gamma)$. In other words, Eq. (13) becomes

$$
\begin{array}{r}
C\left(\bar{A} \oplus B \bar{F}_{o p t}\right)^{*} \widetilde{B}\left(\begin{array}{l}
V(\gamma) \\
Q(\gamma)
\end{array}\right)=C \bar{A}^{*} B V(\gamma) \oplus C \bar{A}^{*} S Q(\gamma), \\
=C \bar{A}^{*} B P_{o p t} Q(\gamma) \oplus C \bar{A}^{*} S Q(\gamma)=C \bar{A}^{*} B P_{o p t} Q(\gamma) \\
=C\left(\bar{A} \oplus B \bar{F}_{\text {opt }}\right)^{*} B P_{o p t} Q(\gamma)=C\left(\bar{A} \oplus B \bar{F}_{\text {opt }}\right)^{*} B V(\gamma) .
\end{array}
$$

Hence, we can achieve the following equality

$$
\begin{array}{r}
C\left(\bar{A} \oplus B \bar{F}_{o p t}\right)^{*} B V(\gamma) \oplus C\left(\bar{A} \oplus B \bar{F}_{o p t}\right)^{*} S Q(\gamma) \\
=C\left(\bar{A} \oplus B \bar{F}_{\text {opt }}\right)^{*} B V(\gamma) .
\end{array}
$$

Therefore, such an integrated control $U(\gamma)$ solves the DDP.

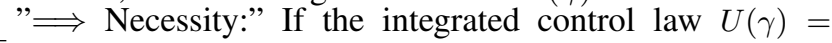
$\bar{F}_{\text {opt }} X(\gamma) \oplus P_{\text {opt }} Q(\gamma)$ solves the DDP, then the following equality holds

$$
\begin{aligned}
C\left(\bar{A} \oplus B \bar{F}_{o p t}\right)^{*} B V(\gamma) & \oplus C\left(\bar{A} \oplus B \bar{F}_{o p t}\right)^{*} S Q(\gamma) \\
= & C\left(\bar{A} \oplus B \bar{F}_{\text {opt }}\right)^{*} B V(\gamma),
\end{aligned}
$$

for an arbitrary disturbance $Q(\gamma)$ and an arbitrary control $V(\gamma)$. Because the feedback control $\bar{F}_{\text {opt }}$ preserves the openloop behaviors, the equality above implies

$$
C \bar{A}^{*} B V(\gamma) \oplus C \bar{A}^{*} S Q(\gamma)=C \bar{A}^{*} B V(\gamma),
$$

for $V(\gamma)=P_{o p t} Q(\gamma)$ to solve the DDP, i.e.

$$
C \bar{A}^{*} B P_{o p t} Q(\gamma) \oplus C \bar{A}^{*} S Q(\gamma)=C \bar{A}^{*} B P_{o p t} Q(\gamma)
$$

Based on Theorem 1, we have $\operatorname{Im} C \bar{A}^{*} S \subset \operatorname{Im} C \bar{A}^{*} B$.

\section{Evaluating the Distance Between Solutions TO DDP AND MDDP IN THE EVENT-DOMAIN}

If the condition $\operatorname{Im} C \bar{A}^{*} S \subset \operatorname{Im} C \bar{A}^{*} B$ in Theorem 1 is not satisfied, we can study the differences between the output trajectories produced by controls solving the MDDP and the output trajectories produced by the disturbances. The differences will give us an evaluation of how much more delays or tokens needed for the controller to the MDDP in order to solve the DDP.

Definition 5: ([17]) Let $X(\gamma)$ and $Y(\gamma)$ be two vector $\gamma$ series with dimensions $n$ and $q$ associate to dater function $k \mapsto x(k)$ and $k \mapsto y(k)$. The residuation

$$
X(\gamma) \phi Y(\gamma)=\bigoplus_{U(\gamma) \in \overline{\mathbb{Z}}_{\max } \llbracket \gamma \rrbracket}\{U(\gamma) \mid U(\gamma) Y(\gamma) \preceq X(\gamma)\}
$$


is named as the correlation matrix of $X(\gamma)$ over $Y(\gamma)$. if $X(\gamma)=Y(\gamma)$, then the residuation $X(\gamma) \phi Y(\gamma)$ is called the autocorrelation matrix of $X(\gamma)$.

The residuation $X(\gamma) \phi Y(\gamma)$ allows us to evaluate the distances between trajectories $X(\gamma)$ and $Y(\gamma)$ in the eventdomain, or in the time-domain if we consider the second order theory in ([17]).

Definition 6: ([19]) Let $x(\gamma)=\oplus_{k \in \mathbb{Z}} x(k) \gamma^{k}$ and $y(\gamma)=$ $\oplus_{k \in \mathbb{Z}} y(k) \gamma^{k}$ be two scalar $\gamma$-series associate to dater function $k \mapsto x(k)$ and $k \mapsto y(k)$. We denote $\mathcal{C}_{x}(t)$ and $\mathcal{C}_{y}(t)$ as the counter function associated to $x(\gamma)$ and $y(\gamma)$, i.e. $x(\gamma)=$ $\oplus_{t \in \mathbb{Z}} t \gamma^{\mathcal{C}_{x}(t)}$, and $y(\gamma)=\oplus_{t \in \mathbb{Z}} t \gamma^{\mathcal{C}_{y}(t)}$. Distance in the eventdomain is defined by

$$
\begin{aligned}
\Delta_{X Y} & =\max \left\{\left|\mathcal{C}_{x}(t)-\mathcal{C}_{y}(t)\right| \text { s.t. } t \in \mathbb{Z}\right\} \\
& =\mathcal{C}_{(x \wedge y) \phi(x \oplus y)}(0) .
\end{aligned}
$$

The last term $\mathcal{C}_{(x \wedge y) \phi(x \oplus y)}(0)$ is the tightest constant upper bound for the tokens in the timed-event graphs.

Proposition 4: Let $x_{1}(\gamma)=s_{1}(\gamma) u(\gamma)$ and $x_{2}(\gamma)=$ $s_{2}(\gamma) u(\gamma)$ two scalar $\gamma$-series describing the behaviors of two states for max-plus linear systems. The distance between these two trajectories in the event-domain is bounded by the distance between the two transfer function series, that is,

$$
\begin{aligned}
\Delta_{x_{1} x_{2}} & =\mathcal{C}_{\left(x_{1} \wedge x_{2}\right) \phi\left(x_{1} \oplus x_{2}\right)}(0) \\
& \leq \Delta_{s_{1} s_{2}}=\mathcal{C}_{\left(s_{1} \wedge s_{2}\right)} \phi\left(s_{1} \oplus s_{2}\right) \\
& (0),
\end{aligned}
$$

for any input $u(\gamma)$.

Proof: Based on the residuation theory in [1] as well as Appendix, we have the following derivations:

$$
\begin{aligned}
& \left(s_{1} u \wedge s_{2} u\right) \phi\left(s_{1} u \oplus s_{2} u\right) \\
& =\left[\left(s_{1} u \wedge s_{2} u\right) \phi s_{1} u\right] \wedge\left[\left(s_{1} u \wedge s_{2} u\right) \phi s_{2} u\right] \\
& (\text { due to }(\mathrm{f} .18)) \\
& =\left[\left(s_{1} u \phi s_{1} u\right) \wedge\left(s_{2} u \phi s_{1} u\right)\right] \wedge\left[\left(s_{1} u \phi s_{2} u\right) \wedge\left(s_{2} u \phi s_{2} u\right)\right] \\
& (\text { due to }(\mathrm{f} .17)) \\
& =\left[\left(\left(s_{1} u \phi u\right) \phi s_{1}\right) \wedge\right. \\
& \left.\left(\left(s_{2} u \phi u\right) \phi s_{1}\right)\right] \wedge\left[\left(\left(s_{1} u \phi u\right) \phi s_{2}\right) \wedge\left(\left(s_{2} u \phi u\right) \phi s_{2}\right)\right] \\
& (\text { due to }(\mathrm{f} .19)) \\
& \succeq\left[\left(s_{1} \phi s_{1}\right) \wedge\left(s_{2} \phi s_{1}\right)\right] \wedge\left[\left(s_{1} \phi s_{2}\right) \wedge\left(s_{2} \phi s_{2}\right)\right] \\
& (\text { due to }(\mathrm{f} .15)) \\
& =\left[\left(s_{1} \wedge s_{2}\right) \phi s_{1}\right] \wedge\left[\left(s_{1} \wedge s_{2}\right) \phi s_{2}\right] \\
& (\text { due to }(\mathrm{f} .17)) \\
& =\left(s_{1} \wedge s_{2}\right) \phi\left(s_{1} \oplus s_{2}\right),(\text { due to }(\mathrm{f} .18)) .
\end{aligned}
$$

Hence, $\forall u(\gamma)$, the following inequality holds:

$$
\left(s_{1} u \wedge s_{2} u\right) \phi\left(s_{1} u \oplus s_{2} u\right) \succeq\left(s_{1} \wedge s_{2}\right) \phi\left(s_{1} \oplus s_{2}\right),
$$

which implies directly:

$$
\mathcal{C}_{\left(x_{1} \wedge x_{2}\right) \phi\left(x_{1} \oplus x_{2}\right)}(0) \leq \mathcal{C}_{\left(s_{1} \wedge s_{2}\right) \phi\left(s_{1} \oplus s_{2}\right)}(0)
$$

for any input $u(\gamma)$.

Theorem 2: ([17] Increasing Correlation Principle) Let $X_{1}(\gamma)=S(\gamma) U(\gamma)$ and $X_{2}(\gamma)=S(\gamma) V(\gamma)$ be the output vectors corresponding to input vectors $U(\gamma)$ and $V(\gamma)$, then

$$
\begin{aligned}
X_{1} \phi X_{2} & \succeq(V \phi U)(S \phi S), \\
X_{1} \phi X_{2} & \succeq(U \phi V) \operatorname{Tr}^{\wedge}(S \phi S),
\end{aligned}
$$

where for any matrix $A$, the dual trace $\operatorname{Tr}^{\wedge}(A)$ is defined as $\operatorname{Tr}^{\wedge}(A)=\bigwedge_{i}(A)_{i i}$

We can use the Increasing Correlation Principle to generate the preceding results to matrix forms of transfer series.
Theorem 3: Let $X_{1}(\gamma)=S_{1}(\gamma) U(\gamma)$ and $X_{2}(\gamma)=$ $S_{2}(\gamma) U(\gamma)$ be the output vectors corresponding to the same input vector $U(\gamma)$, then

$$
\begin{aligned}
& X_{1} \phi X_{2} \succeq S_{1} \phi S_{2}, \\
& X_{1} \phi X_{2} \succeq \operatorname{Tr}^{\wedge}\left(S_{2} \phi S_{1}\right),
\end{aligned}
$$

where $\operatorname{Tr}^{\wedge}\left(S_{2} \phi S_{1}\right)=\bigwedge_{i}\left(S_{2} \phi S_{1}\right)_{i i}$.

Proof: Based on the residuation theory in ([1]), we have the following derivations:

$$
\begin{aligned}
X_{1} \phi X_{2} & =\left(S_{1} U\right) \phi\left(S_{2} U\right) \\
& =\left(\left(S_{1} U\right) \phi U\right) \phi S_{2}, \text { due to (f.19) } \\
& \succeq S_{1} \phi S_{2}, \text { due to (f.15) }
\end{aligned}
$$

and

$$
\begin{aligned}
X_{1} \phi X_{2}= & \left(S_{1} U\right) \phi\left(S_{2} U\right) \\
= & U \phi\left(S_{1} \phi\left(S_{2} U\right)\right), \text { due to (f.11) } \\
\succeq & U \phi\left(\left(S_{1} \phi S_{2}\right) U\right), \text { due to }(\mathrm{f} .13) \\
\succeq & U \phi\left[\left(\operatorname{Tr}^{\wedge}\left(S_{2} \phi S_{1}\right)\right) U\right], \\
& \text { due to } \operatorname{Tr}^{\wedge}\left(S_{2} \phi S_{1}\right)=S_{1} \phi S_{2}, \\
\succeq & (U \phi U) \operatorname{Tr}^{\wedge}\left(S_{2} \phi S_{1}\right), \\
& \quad \text { due to } \operatorname{Tr}^{\wedge}\left(S_{2} \phi S_{1}\right) \text { is scalar, } \\
\succeq & \operatorname{Tr}^{\wedge}\left(S_{2} \phi S_{1}\right) \\
& \text { due to } U \phi U \succeq \text { Id. }
\end{aligned}
$$

In the remaining section, we will apply the preceding results in order to compare the difference between the output trajectories induced by controls and by disturbances, i.e, the distance between $Y_{1}=C \bar{A}^{*} B P_{o p t} Q(\gamma)$, and $Y_{2}=C \bar{A}^{*} S Q(\gamma)$. The two output trajectories are obviously ordered because $P_{\text {opt }}=\left(C \bar{A}^{*} B\right) \phi\left(C \bar{A}^{*} S\right)$, i.e. $Y_{1} \preceq Y_{2}$, which solves for the MDDP. On the other hand, if $Y_{1}=Y_{2}$, then the DDP is solved. The question is that, if $Y_{1} \neq Y_{2}$, then how much is the distance between the two output trajectories.

Proposition 5: Let $Y_{1}(\gamma)=C \bar{A}^{*} B P_{o p t} Q(\gamma)$ and $Y_{2}(\gamma)=$ $C \bar{A}^{*} S Q(\gamma)$ two $\gamma$-series describing the output behaviors of the max-plus linear systems in Eq. (3), where $P_{o p t}=$ $\left(C \bar{A}^{*} B\right) \phi\left(C \bar{A}^{*} S\right)$. The distance between these two output trajectories in the event domain is bounded by the distance between the two transfer function series, that is,

$$
\Delta_{Y_{1} Y_{2}}=\mathcal{C}_{Y_{1} \phi Y_{2}}(0) \leq \mathcal{C}_{\left(C \bar{A}^{*} B P_{\text {opt }}\right) \phi\left(C \bar{A}^{*} S\right)}(0),
$$

for any distance input $Q(\gamma)$.

Proof: Using Eq. (23) in Theorem 3, we can obtain that $Y_{1} \phi Y_{2}=\left(C \bar{A}^{*} B P_{\text {opt }} Q\right) \phi\left(C \bar{A}^{*} S Q\right) \succeq\left(C \bar{A}^{*} B P_{\text {opt }}\right) \phi\left(C \bar{A}^{*} S\right)$. Therefore, the distance between these two output trajectories in the event-domain satisfies the following equality

$$
\Delta_{Y_{1} Y_{2}}=\mathcal{C}_{Y_{1} \phi Y_{2}}(0) \leq \mathcal{C}_{\left(C \bar{A}^{*} B P_{\text {opt }}\right) \phi\left(C \bar{A}^{*} S\right)}(0)
$$

VI. Application to A High Throughrut Screening SYSTEM IN DRUG DISCOVERY

High throughput screening (HTS) is a standard technology in drug discovery. In HTS systems, optimal scheduling is required to finish the screening in the shortest time, as well as to preserve the consistent time spending on each activity. This section is using a HTS system to illustrate the main results in this paper. This HTS system, adapted from [3], has three nested activities running on three different singlecapacity resources: pipettor (activity 1), reader (activity 2), and incubator (activity 3). The Gantt chart for this HTS system is shown in Fig. 2. One cycle of events is shown as follows: first, the pipettor drops the DNA/RNA compounds into the microplate, then the microplate is transferred to the reader to be scanned, and then the microplate is transferred to the incubator to develop. After the first cycle of events, the 
second cycle of event will start. Moreover, the three activities are overlapping during the transition time, for instance, the reader starts scanning 3 time units before the pipettor finishes its task, and finishes scanning 7 units after incubator starts the task, as shown in Fig. 2.

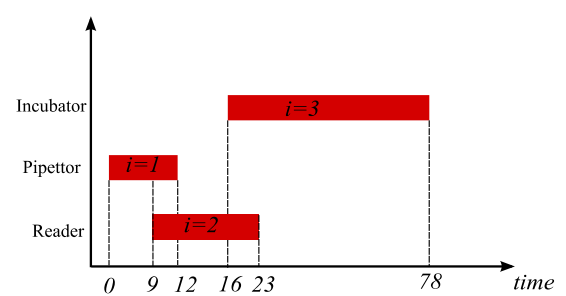

Fig. 2: The Gantt chart of one cycle of activities.

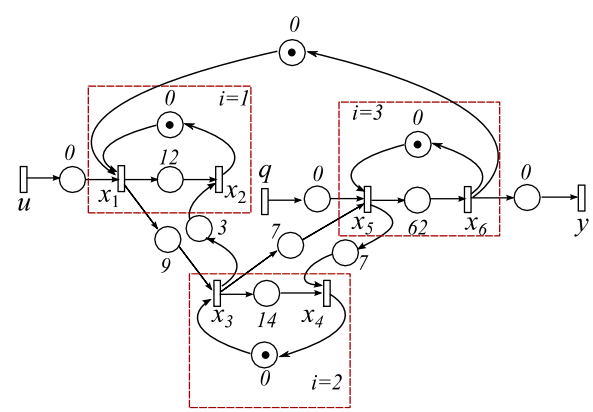

Fig. 3: The TEG model for the HTS system.

If we are interested in the start and release event time of each activity, we can model the HTS system as a TEG model, shown in Fig. 3, in which $x_{1}$ and $x_{2}$ denote the start and release time of the activity. 1 on the pipettor, $x_{3}$ and $x_{4}$ denote the start and release time of the activity 2 on the reader, and $x_{5}$ and $x_{6}$ denote the start and release time of the activity 3 on the incubator. The input $u$ is the starting time of the pipettor which users can decide when to load the chemical compounds. The disturbance $q$ is the starting time of the incubator, such as transition time delay from the reader to the incubator due to system malfunction. The output $y$ is the release time of the incubator. The cycles represent places and the bars represent the transitions $x_{i}$. The tokens in the places represent that the transitions are ready to be fired, i.e. the activity is ready to start. For the TEG model of a HTS system shown in Fig. 3, the system over the max-plus algebra $\overline{\mathbb{Z}}_{\max } \llbracket \gamma \rrbracket$ is described as the following:

$$
\begin{aligned}
X(\gamma) & =\bar{A} X(\gamma) \oplus B U(\gamma) \oplus S Q(\gamma) \\
Y(\gamma) & =C X(\gamma), \text { where } \\
\bar{A} & =\left[\begin{array}{cccccc}
\varepsilon & \gamma & \varepsilon & \varepsilon & \varepsilon & \gamma \\
12 & \varepsilon & 3 & \varepsilon & \varepsilon & \varepsilon \\
9 & \varepsilon & \varepsilon & \gamma & \varepsilon & \varepsilon \\
\varepsilon & \varepsilon & 14 & \varepsilon & 7 & \varepsilon \\
\varepsilon & \varepsilon & 7 & \varepsilon & \varepsilon & \gamma \\
\varepsilon & \varepsilon & \varepsilon & \varepsilon & 62 & \varepsilon
\end{array}\right], \\
B^{T} & =\left[\begin{array}{llllll}
e & \varepsilon & \varepsilon & \varepsilon & \varepsilon & \varepsilon
\end{array}\right], \\
S^{T} & =\left[\begin{array}{llllll}
\varepsilon & \varepsilon & \varepsilon & \varepsilon & e & \varepsilon
\end{array}\right], \text { and } \\
C & =\left[\begin{array}{llllll}
\varepsilon & \varepsilon & \varepsilon & \varepsilon & \varepsilon & e
\end{array}\right] .
\end{aligned}
$$

The example has been computed by using toolbox MinMaxGD, a C++ library allowing to handle periodic series as introduced in ([5]), and it can be noted that this library is also interfaced with Scilab and MATLAB. We obtain The transfer function between the output $Y(\gamma)$ and disturbance $U(\gamma)$ and the input $Q(\gamma)$, respectively, as

$$
\begin{aligned}
C \bar{A}^{*} B & =78(78 \gamma)^{*}=78 \oplus 156 \gamma \oplus 234 \gamma^{2} \cdots, \\
C \bar{A}^{*} S & =62(78 \gamma)^{*}=62 \oplus 140 \gamma \oplus 218 \gamma^{2} \cdots,
\end{aligned}
$$

in which each component of these matrices is a periodic series. Essentially, the $\gamma$-periodic series represent the output sequence when an infinity of inputs is put in the system at time 0 (impulse input). For instance, $C \bar{A}^{*} B$ represents the impulse response of the incubator as $y(0)=78, y(1)=156$, $y(2)=234$, etc.

The non-causal filter $P_{\text {opt }}$ is $\left(C \bar{A}^{*} B\right) \phi\left(C \bar{A}^{*} S\right)=$ $-16(78 \gamma)^{*}$. In this example, we have $\operatorname{Im} C \bar{A}^{*} S_{0} \subset \operatorname{Im} C \bar{A}^{*} B_{0}$, hence, this non-causal prefilter solves the MDDP and the DDP at the same time due to $C \bar{A}^{*} B P_{o p t}=C \bar{A}^{*} S$. This prefilter $P_{o p t}$ is not causal because there are negative coefficients in the matrix. The canonical injection from the causal elements of $\overline{\mathbb{Z}}_{\max } \llbracket \gamma \rrbracket$ (denoted $\overline{\mathbb{Z}}_{\max } \llbracket \gamma \rrbracket^{+}$) in $\overline{\mathbb{Z}}_{\max } \llbracket \gamma \rrbracket$ is also residuated (see [6] for details). Its residual is given by $\operatorname{Pr}\left(\bigoplus_{k \in \mathbb{Z}} s(k) \gamma^{k}\right)=\bigoplus_{k \in \mathbb{Z}} s_{+}(k) \gamma^{k}$ where

$$
s_{+}(k)=\left\{\begin{array}{l}
s(k) \text { if }(k, s(k)) \geq(0,0), \\
\varepsilon \text { otherwise. }
\end{array}\right.
$$

If we take the canonical injection from the causal elements of $\overline{\mathbb{Z}}_{\max } \llbracket \gamma \rrbracket$, then the greatest causal prefilter is

$$
P_{\text {opt }+}=\operatorname{Pr}\left(P_{\text {opt }}\right)=62 \gamma(78 \gamma)^{*} \text {. }
$$

The causal filter $P_{o p t+}$ for the MDDP does not solve DDP because

$$
C \bar{A}^{*} B P_{\text {opt }+}=140 \gamma(78 \gamma)^{*} \prec C \bar{A}^{*} S .
$$

Using Proposition 5, we can evaluate the difference between the output trajectories produced by the pre-filter $P_{o p t+}$ and by the disturbances only. The distance is

$$
\begin{aligned}
\left(C \bar{A}^{*} B P_{o p t+}\right) \phi\left(C \bar{A}^{*} S\right) & =78 \gamma(78 \gamma)^{*} \\
\Delta_{Y_{1} Y_{2}} & \leq \mathcal{C}_{\left(C \bar{A}^{*} B P_{\text {opt }}\right) \phi\left(C \bar{A}^{*} S\right)}(0)=1 .
\end{aligned}
$$

This distance indicates that there is one event delay between output trajectories produced by the pre-filter $P_{o p t+}$ and by the disturbances only.

According to Proposition 1 and Eq. (14), the greatest feedback controller preserving the open-loop behaviors is obtained as follows:

$$
\begin{aligned}
\bar{F}_{o p t}= & \left(C \bar{A}^{*} B\right) \phi\left(C \bar{A}^{*} \bar{B}\right) \phi\left(\bar{A}^{*} \bar{B}\right) \\
= & {\left[(78 \gamma)^{*},-12(78 \gamma)^{*},-9(78 \gamma)^{*},\right.} \\
& \left.-23(78 \gamma)^{*},-16(78 \gamma)^{*},-78(78 \gamma)^{*}\right] .
\end{aligned}
$$

The greatest causal feedback is

$$
\begin{aligned}
\bar{F}_{o p t+}= & \operatorname{Pr}\left(\bar{F}_{o p t}\right)=\left[(78 \gamma)^{*}, 66 \gamma(78 \gamma)^{*}, 69 \gamma(78 \gamma)^{*},\right. \\
& \left.55 \gamma(78 \gamma)^{*}, 62 \gamma(78 \gamma)^{*}, \gamma(78 \gamma)^{*}\right] .
\end{aligned}
$$

The TEG model of the HTS system with open-loop and state feedback controllers are shown in Fig. 4.

In Fig. 4, the causal pre-filter $P_{\text {opt }+}=62 \gamma(78 \gamma)^{*}$ is represented by a cyclic transition due to $(78 \gamma)^{*}$, where $\gamma$ indicates one token inside of the place and 78 units are the holding time of the token, and $62 \gamma$ is represented by an additional transition with one token and 62 units holding time. Same analogy works for the feedback $\bar{F}_{\text {opt }+}: X \rightarrow U$.

When we apply the integrated control law $U(\gamma)=$ $\bar{F}_{\text {opt+ }} X(\gamma) \oplus P_{\text {opt }} Q(\gamma)$ to the system, we can solve the MDDP and the DDP because

$C\left(\bar{A} \oplus B \bar{F}_{o p t+}\right)^{*} B P_{o p t}=C\left(\bar{A} \oplus B \bar{F}_{o p t+}\right)^{*} S=C \bar{A}^{*} S$, 


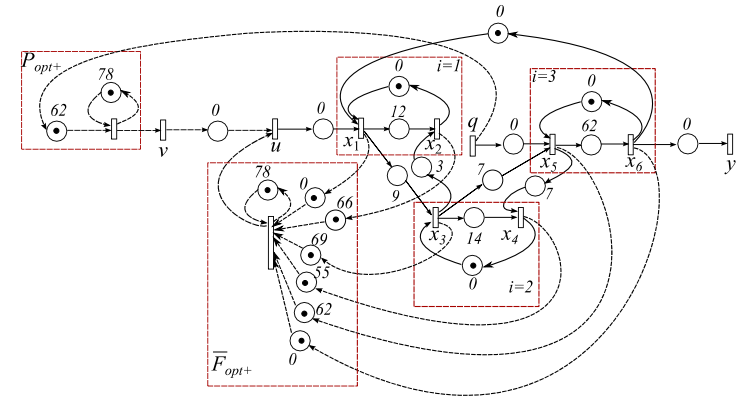

Fig. 4: The TEG model of the HTS system with controllers.

i,e, equations (10) and (11) are both satisfied. On the other hand, when When we apply the integrated control law $U(\gamma)=\bar{F}_{o p t+} X(\gamma) \oplus P_{o p t+} Q(\gamma)$ to the system instead, we can only solve the MDDP, but not for the DDP because

$C\left(\bar{A} \oplus B \bar{F}_{\text {opt+ }}\right)^{*} B P_{o p t+}=140 \gamma(78 \gamma)^{*} \prec C\left(\bar{A} \oplus B \bar{F}_{\text {opt+ }}\right)^{*} S$,

i.e. only Eq. (11) is satisfied, but Eq. (10) is not. In this case, we can evaluate the distance between the two output trajectories of the state-feedback controlled system and of the uncontrolled system. Using Proposition 5, we obtain that

$$
\begin{array}{r}
\left(C\left(\bar{A} \oplus B \bar{F}_{\text {opt+ }}\right)^{*} B P_{o p t+}\right) \phi\left(C\left(\bar{A} \oplus B \bar{F}_{o p t+}\right)^{*} S\right)=78 \gamma(78 \gamma)^{*} \\
\Delta_{Y_{1} Y_{2}} \leq \mathcal{C}_{\left(C\left(\bar{A} \oplus B \bar{F}_{o p t+}\right)^{*} B P_{o p t+}\right) \phi\left(C\left(\bar{A} \oplus B \bar{F}_{o p t+}\right)^{*} S\right)}(0)=1 .
\end{array}
$$

\section{CONCLUSIONS}

This paper presents the integration of the state-feedback controls and the open-loop controls to solve the MDDP, as well as the DDP. If these controls can only solve the MDDP, not the DDP, an evaluation principle is established such that the performance of the controls solving the MDDP can be evaluated. Future research can be extended to other geometric control problems, such as block decoupling problem and non-interacting control problem in max-plus linear systems.

\section{REFERENCES}

[1] F. Baccelli, G. Cohen, G.J. Olsder, and J.-P. Quadrat, Synchronization and Linearity: An Algebra for discrete-event Systems. New York: John Wiley and Sons, 1992. http://www-rocq.inria.fr/ metalau/cohen/SED/SED1-book.html

[2] G. Basile and G. Marro, "Controlled and conditioned invariant subspaces in linear system theory", Journal of Optimization Theory and Applications, volume $3,5,1969$, pp. 306-315.

[3] T. Brunsch, J. Raisch, and L. Hardouin, "Modeling and control of high-throughput screening systems", Control Engineering Practice, vol. 20, 2012, 14-23.

[4] G. Cohen, S. Gaubert, and J.-P. Quadrat. "Max-plus algebra and system theory: where we are and where to go now", Annual Reviews in Control, vol. 23, 1999, pp. 207-219.

[5] B. Cottenceau, L. Hardouin, M. Lhommeau, and J.-L. Boimond, "Data processing tool for calculation in dioid", The IEEE International Workshop on discrete-event Systems, Ghent, Belgium, 2000. http: // WwW. istia.univ-angers. fr/ hardouin/outils.html

[6] B. Cottenceau, L. Hardouin, J.-L. Boimond, and J.-L. Ferrier, "Model reference control for timed-event graphs in dioid", Automatica, vol. 37, 2001, pp. 1451-1458.

[7] L. Hardouin, C.A. Maia, B. Cottenceau, and M. Lhommeau, "Observer Design for (max,plus)-Linear Systems", IEEE Transactions on Automatic Control, vol. 5, issue 2, 2010, pp. 538-543.

[8] L. Hardouin, M. Lhommeau, and Y. Shang, "Towards Geometric Control of Max-Plus Linear Systems with Applications to Manufacturing Systems", The 50th IEEE Conference on Decision and Control and European Control Conference, Orlando, FL. USA, 2011, pp. 11491154.

[9] R.D. Katz, "Max-plus (A,B)-invariant spaces and control of timed discrete-event systems", IEEE Transactions on Automatic Control, vol. 52, issue 2, 2007, pp. 229-241.

[10] J.-Y. Le Boudec, and P. Thiran, Network Calculus. New York: Springer-Verlag, 2002.
[11] M. Lhommeau, L. Hardouin and B. Cottenceau, "Disturbance decoupling of timed-event graphs by output feedback controller", The 6th International Workshop on discrete-event Systems, Zaragoza, Spain, 2002. http://www.istia.univ-angers. $\mathrm{fr} / \sim$ hardouin/Wodes 02 .pdf

[12] M. Lhommeau, L. Hardouin, and B. Cottenceau, "About disturbance decoupling of timed-event graphs in dioids", The 6th International Workshop on Discrete Event Systems, Zaragoza, Spain, 203-208, 2002. http: //www. istia.univ-angers.fr/ $\sim$ hardouin/Wodes 02 .pdf

[13] M. Lhommeau, L. Hardouin and B. Cottenceau, O"ptimal control for (max,+)-linear systems in the presence of disturbances", Positive Systems: Theory and Applications, POSTA, Springer LNCIS 294,2003, pp. $47-54$.

[14] M. Di Loreto, S. Gaubert, R. Katz, and J-J. Loiseau, "Duality, between invariant spaces for max-plus linear discrete-event systems", SIAM Journal on Control and Optimization, vol. 48, 2010, pp.5606-5628. http://fr.arXiv.org/abs/0901.2915.

[15] C.A. Maia, L. Hardouin, R. Santos-Mendes, and B. Cottenceau, "On the Model reference control for max-plus linear systems", The 44th IEEE Conference on Decision and Control, 2005, pp. 7799-7803.

[16] C.A. Maia, C.R. Andrade, and L. Hardouin,newblock "On the control of max-plus linear system subject to state restriction", Automatica, vol.47, issue 5, 2011, pp. 988-992.

[17] MaxPlus Group "Second order theory of min-linear systems and its application to discrete-event systems", Proceedings of the 30th IEEE Conference on Decision and Control, 1991.

[18] J.-M. Prou and E. Wagneur.(1999). Controllability in the max-algebra. KYBERNETIKA, vol. 35, issue 1, 1999, pp.13-24.

[19] R. Santos-Mendes, B. Cottenceau, and L. Hardouin, "Adaptive feedback control for (max,+)-linear systems", The 10th IEEE International Conference on Emerging Technologies and Factory Automation, vol. 2, 2005, pp. 164-170. http://dx.doi.org/10.1109/ ETFA.2005.1612675

[20] Y. Shang, L. Hardouin, M. Lhommeau and C.A. Maia. "Open-loop controllers to solve the disturbance decoupling problem for maxplus linear systems", 2013 European Control Conference, Zurich, Switzerland, 2013, pp. 125-130.

[21] W.M. Wonham, Linear Multivariable Control: A Geometric Approach. New York: Springer-Verlag, 1979.

\section{APPENDIX}

\section{A. Formulas of Star Operations}

\begin{tabular}{cc}
\hline$a^{*}\left(b a^{*}\right)^{*}=(a \oplus b)^{*}=\left(a^{*} b\right)^{*} a^{*}$ & (f.1) \\
$\left(a^{*}\right)^{*}=a^{*}$ & (f.2) \\
$(a b)^{*} a=a(b a)^{*}$ & (f.3) \\
$a^{*} a^{*}=a^{*}$ & (f.4) \\
$a a^{*}=a^{*} a$ & (f.5) \\
\hline
\end{tabular}

\section{B. Formulas of Left Residuations}

\begin{tabular}{cc}
\hline$a(a \phi x) \preceq x$ & (f.6) \\
$a \phi(a x) \succeq x$ & (f.7) \\
$a(a \phi(a x))=a x$ & (f. 8$)$ \\
$a \phi(x \wedge y)=a \phi x \wedge a \phi y$ & (f.9) \\
$(a \oplus b) \phi x=a \phi x \wedge b \phi x$ & (f.10) \\
$(a b) \phi x=b \phi(a \phi x)$ & (f.11) \\
$b(a \phi x) \preceq(a \phi b) \phi x$ & (f.12) \\
$(a \phi x) b \preceq a \phi(x b)$ & (f.13) \\
\hline
\end{tabular}

\section{Formulas of Right Residuations}

\begin{tabular}{cc}
\hline$(x \phi a) a \preceq x$ & (f.14) \\
$(x a) \phi a \succeq x$ & (f.15) \\
$((x a) \phi a) a=x a$ & (f.16) \\
$(x \wedge y) \phi a=x \phi a \wedge y \phi a$ & (f.17) \\
$x \phi(a \oplus b)=x \phi a \wedge x \phi b$ & (f.18) \\
$x \phi(b a)=(x \phi a) \phi b$ & (f.19) \\
$(x \phi a) b \preceq x \phi(b \phi a)$ & (f.20) \\
$b(x \phi a) \preceq(b x) \phi a$ & (f.21) \\
\hline
\end{tabular}

